Research Article

\title{
The Value of Convolutional-Neural-Network-Algorithm-Based Magnetic Resonance Imaging in the Diagnosis of Sports Knee Osteoarthropathy
}

\author{
Rong Yan \\ Anhui Medical College, Hefei 230601, Anhui, China \\ Correspondence should be addressed to Rong Yan; yanrong@ahyz.edu.cn
}

Received 10 May 2021; Revised 14 June 2021; Accepted 26 June 2021; Published 3 July 2021

Academic Editor: Gustavo Ramirez

Copyright (c) 2021 Rong Yan. This is an open access article distributed under the Creative Commons Attribution License, which permits unrestricted use, distribution, and reproduction in any medium, provided the original work is properly cited.

\begin{abstract}
The application value of the convolutional neural network (CNN) algorithm in the diagnosis of sports knee osteoarthropathy was investigated in this study. A network model was constructed in this experiment for image analysis of magnetic resonance imaging (MRI) technology. Then, 100 cases of sports knee osteoarthropathy patients and 50 healthy volunteers were selected. Digital radiography (DR) images and MRI images of all the research objects were collected after the inclusion of the two groups. Besides, the important physiological representations were extracted from their image data graphs, and the hidden complex relationships were learned. The state without input results was judged through convolutional network calculation, and the result prediction was given. On this basis, there was an analysis of the diagnostic efficiency of traditional DR images and MRI images based on CNN for patients with sports knee osteoarthropathy. The results showed that the MRI images analyzed by the CNN model showed a more obvious display rate than DR images for some nonbone changes of osteoarthritis. The correlation coefficient between MRI image rating and visual analog scale (VAS) was 0.865 , which was higher than 0.713 of DR image rating, with a statistical meaning $(P<0.01)$. For cases with mild lesions, the number of cases detected by MRI based on CNN algorithm in $0-4$ image rating was 15 , $18,10,6$, and 7, respectively, which was markedly better than that of DR images. In short, the MRI examination based on the CNN image analysis model could extract important physiological representations from the image data and learn the hidden complex relationships. The convolutional network was calculated to determine the state of the uninput results and give the result predictions. Moreover, MRI examination based on the CNN image analysis model had high overall diagnostic efficiency and grading diagnostic efficiency for patients with motor knee osteoarthropathy, which was of great significance in clinical practice.
\end{abstract}

\section{Introduction}

Sports knee osteoarthropathy is a type of disease caused by degenerative lesions of the knee joints, external force injuries, overwork, and other reasons. Clinically, it is usually manifested as swelling and pain in the knee, difficulty in going upstairs and downstairs, and fluid in the knee [1]. In the knee joints, patients often suffer from joint diseases such as joint capsule disease, knee cartilage degeneration, and other joint diseases. Many patients with sports knee osteoarthropathy have mild early clinical symptoms, and their condition will worsen if they are not paid attention to and receive treatment.

Clinically, pathological judgments are usually based on imaging examinations. In imaging examinations, the most commonly used methods are DR and MRI, and these two methods are mostly used for pathological diagnosis of sports knee osteoarthropathy in clinical practice. Among them, DR image can show bone structural changes such as proliferative osteoarthropathy, knee joint space narrowing, and subchondral ossification, but it cannot display the hyaline cartilage or fibrocartilage. Besides, the examination of the initial changes of knee cartilage tissue also lacks sensitivity and is not suitable for the early diagnosis of sports knee osteoarthropathy [2]. MRI examination plays a more obvious role in diseases such as structural changes of knee bone and joint. It can display images from multiple azimuthal angles and help physicians to better understand the structure of joint bone, joint, and joint soft tissue, with high accuracy, 
specificity, and sensitivity [3]. However, MRI examination report has a high requirement for manual film reading, and nonexperienced doctors cannot accurately explore and judge it. In addition, there are many types of clinical classification of sports knee osteoarthropathy, and there are many similar characteristics among them. These image data are manually processed by clinicians so that the time cost is greatly increased, and the quality of pathological analysis and diagnosis will also be affected [4]. In recent years, artificial intelligence methods have occupied a critical position in the development and application of medical imaging circles, and assisting in the diagnosis of clinical-pathological imaging is of great significance to clinical diagnosis. However, there are some challenges and difficulties that need to be solved in analyzing pathology images through computers: pathology images are often large in size and high in resolution. At present, the hardware processing equipment equipped in some medical institutions at the basic level or in economically underdeveloped areas cannot process the whole pathological image quickly and perfectly, so it is necessary to develop a more advanced, more convenient, and practical algorithm. Since the composition of the pathological image is too complex, it also has higher requirements for the algorithm.

CNN algorithm can quickly check, test, and measure the images for classification, thereby improving the classification precision and accuracy and reducing the probability of error diagnosis delay in diagnosis, which can be step by step close to or even better than the clinician experience of diagnosis. It is expected to achieve effect in the treatment of patients and assess the prognosis and other important clinical information [5]. Therefore, the effective features and target information are extracted through the automatic analysis of imaging images, and quantum chemical calculation methods are adopted to obtain quantitative indicators that can be reflected by specific data. When accurate diagnosis results are obtained, automatic calculation and extraction of characteristic information of imaging images are carried out by computer, which greatly improves work efficiency, reduces working hours, and alleviates the workload of clinicians, to avoid errors or imperfections in analysis and diagnosis caused by a heavy workload. In this experiment, the multisequence MRI image sample data of knee cartilage tissue collected were used as the training set to establish the CNN network model, and the diagnostic efficiency of the model for knee cartilage tissue and MRI images was analyzed.

\section{Materials and Methods}

2.1. Research Objects. A total of 100 patients with a clinical diagnosis of sports knee osteoarthropathy (200 knee joints), who were treated in hospital from August 2017 to December 2020 , were collected as the research objects of this experiment, which were set as a lesion group, and all the patients complained of pain in the knee joint. What is more, all patients received DR and MRI examinations, and the general clinical data were complete. Among them, there were 60 males and 40 females, aged $45-75$ years, with an average age of $60 \pm 3.46$ years; the course of the disease was 3 months -5 years, with an average length of $2.6 \pm 0.53$ years. There were 99 with sports knee osteoarthropathy on the left and 101 with sports knee osteoarthropathy on the right. Moreover, 50 healthy people with 100 knee joints who underwent physical examinations in the hospital at the same time were selected as a control group, including 30 males and $20 \mathrm{fe}$ males, aged 46-76 years, with an average age of $61 \pm 3.88$ years. None of them had a history of knee osteoarthralgia, without any clinical symptoms or signs. The criteria for inclusion were defined to include patients who had the clinical manifestations of knee joint pain, met the grade 0 -II of the imaging grading, and had the degeneration and injury of the joint cartilage in the imaging examination. The criteria for exclusion were defined to include patients who suffered from inflammatory joint diseases such as rheumatoid arthritis and gouty arthritis; had autoimmune system diseases, blood system diseases, tuberculosis or tumors, bone biochemical metabolic disorders of bone disease, and other diseases; were accompanied with major organ diseases; were pregnant or in the breastfeeding period; and had senile dementia or mental illness. In addition, all participants and their family members were informed of all matters of this experiment and signed the consent forms.

2.2. DR Examination. Examinations were carried out using a C50 suspension digital X-ray system (produced by Philips, Netherlands). During the examination, the patient first took the supine position, with the legs stretched flat, the knee joints were completely exposed, and an orthographic film was taken. Subsequently, the lateral radiographs were taken in the lateral decubitus position, and the obtained images were exported and stored on the hard disk for later analysis. The main observation indicators included the osteoplasia of knee joint space stenosis and subchondral osteosclerosis.

2.3. MRI Examination. The $1.5 \mathrm{~T}$ superconducting magnetic resonance scanner (produced by Siemens AG, Germany) was used for examination. The patient was placed in a supine position, with both legs and knee joints stretched naturally, and placed in a special coil for the knee joint. Routine general electric (GE) sequences were performed, and sagittal T1-weighted imaging (T1WI), fat-suppression T2WI, magnetic resonance proton imaging, coronal T1WI, and transverse T2WI were adopted in this experiment. When necessary, T1WI enhancement scanning was applied, including sagittal, coronal, and transverse planes, with a layer thickness of $5 \mathrm{~mm}$ and layer spacing of $2 \mathrm{~mm}$. There was the observation mainly for the formation of hyaline cartilage or fibrocartilage in joints, knee joint effusion, articular subchondral bone sclerosis, small subchondral cysts of joint, and joint marginal bone hyperplasia.

2.4. A Model Based on the CNN Algorithm. CNN is a representative model of deep learning (DL) [6], which is a feedforward neural network with a local connection and weight sharing. It achieves weight sharing in time and reduces 
internal parameters, to reduce the complexity and difficulty of computer learning. $\mathrm{CNN}$ has an advantage in recognizing natural images [7]. For natural images with stable local areas, $\mathrm{CNN}$ can learn target features suitable for other adjacent local areas and convolve the images in a certain order [8], to extract one or several features. Therefore, multiple convolution kernels are needed if multiple different features are needed to be extracted, which can reduce the learning parameters of the network.

The composition of CNN is roughly divided into four parts: input layer, convolutional layer, fully connected layer, and output layer, as shown in Figure 1.

The CNN-based network model is a model that is mostly applied in the fields of image classification and distinguishing recognition and has excellent feature extraction functions. The $\mathrm{CNN}$ model designed in this experiment employed the error backpropagation algorithm [9]. First, the parameters inside the network were initialized, and then a sample $Z$ and the corresponding mathematical expectation mean were input to the network. Then, $Q$ was output, and the output of each layer was calculated. Besides, the output of the $m$-th neuron in the $f$-th layer was shown in

$$
\begin{aligned}
U_{m}^{f} & =\sum_{n=1}^{p+1} W_{m n} X_{n}^{f-1}, \quad\left(Z_{p+1}^{f-1}=1, W_{m, p+1}=-\theta\right), \\
Z_{m}^{f} & =K\left(U_{m}^{f}\right) .
\end{aligned}
$$

According to the geometric error cost function, the error of each layer of parameter variables was obtained [10], and the output layer could be expressed in

$$
\begin{aligned}
f & =i: d_{(m)}^{(i)} \\
& =Z_{(m)}^{(i)}\left(1-Z_{m}^{(i)}\right)\left(Z_{m}^{(i)}-Q_{m}\right), \\
d_{m}^{(f)} & =Z_{m}^{(f)}\left(1-Z_{m}^{(f)}\right) \sum_{l} W_{l m} d_{l}^{(f+1)} .
\end{aligned}
$$

Before the CNN model was trained, the parameter variables had to be initialized [11]. If the initialization value stayed in an area where the error was relatively flat, it would cause its convergence speed to become too slow, so more initialization weight parameters were chosen and their distribution was presented in

$$
W \sim U\left[-\frac{\sqrt{5}}{\sqrt{n^{(f)}+n^{(f-1)}}}, \frac{\sqrt{5}}{\sqrt{n^{(f)}+n^{(f-1)}}}\right] .
$$

In $\mathrm{CNN}$, it is necessary to increase the number of layers to enhance the network learning ability. However, if the sample data are too small, the phenomenon of overfitting will occur [12]; thus, the network fails to generalize the prediction of the events to be known to the rest of the data samples. To avoid such problems, a regularization method was applied in this experiment [13], which could retain all the characteristic parameters and only reduce the scale and size of the data volume. In other words, a part describing the complexity of the network was added in the loss function, and the amplitude of the weight parameter was reduced so

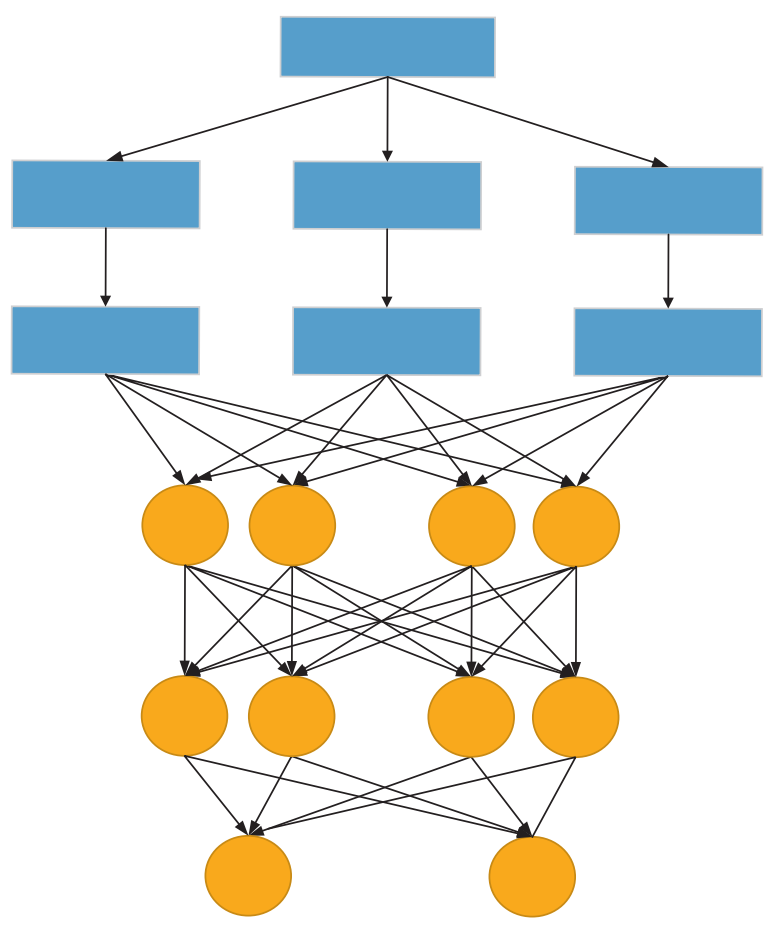

Figure 1: The basic composition of CNN.

that it was more suitable for finite video memory and had the function of efficient image classification. What is more, the structure was shown in Figure 2.

In this experiment, the MRI images of the same part of 100 sports knee osteoarthropathy patients and 50 healthy people without the disease were collected in the previous stage for prediagnosis training, and the sample data graphs of patients one by one were for a series of preprocessing. Figure 3 showed the partial samples of all the training sample data maps of the $\mathrm{CNN}$ model obtained after preprocessing.

Then, 150 MRI images (100 images from patients with sports knee osteoarthropathy and 50 from healthy people without the disease) were selected for the accuracy test. Next, the preprocessed image summary set was stored in a vertical and horizontal array [14], and the vertical and horizontal array and the corresponding binary class label were stored in the data storage file of MATLAB at the same time, which were read into the network. After verification, there were 130 accuracy cases, with an average accuracy of $86.67 \%$. Finally, the patient's image map was read into the CNN model to start the diagnosis of the disease, and the result of the disease prediction was "the patient was suspected of having sports knee osteoarthropathy," and the diagnosis was correct. Compared with computer-assisted diagnosis, the accuracy and performance of the CNN model were better. CNN usually independently carries out image training tests, which are mostly applied in the field of unsupervised learning. The characteristic images obtained at each layer are used as input for deconvolution calculation [15], to obtain the deconvolution results. To verify and display the feature images extracted from each layer, the visual network structure was shown in Figure 4. In 


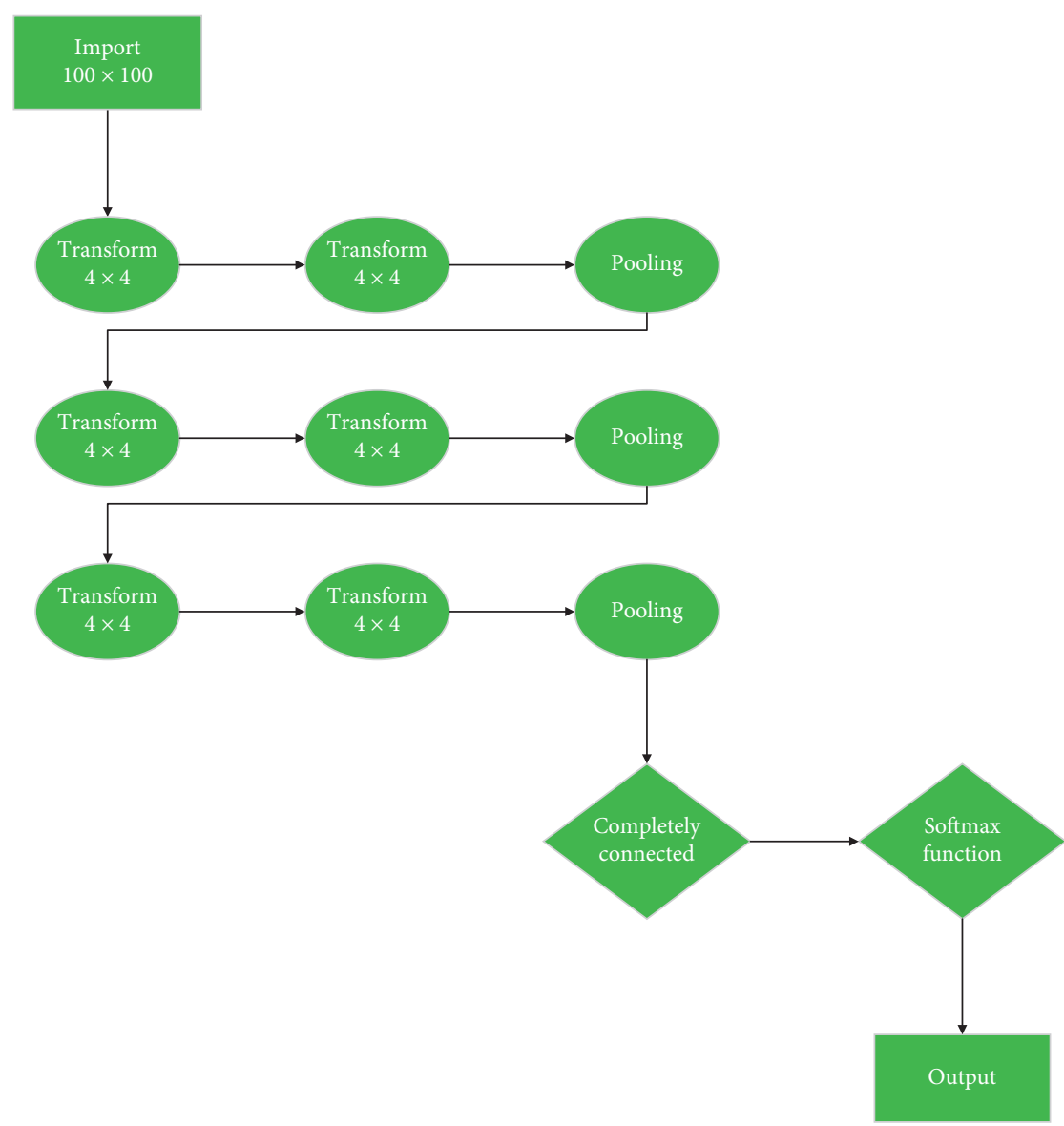

FIgURE 2: Schematic diagram of CNN network model structure.

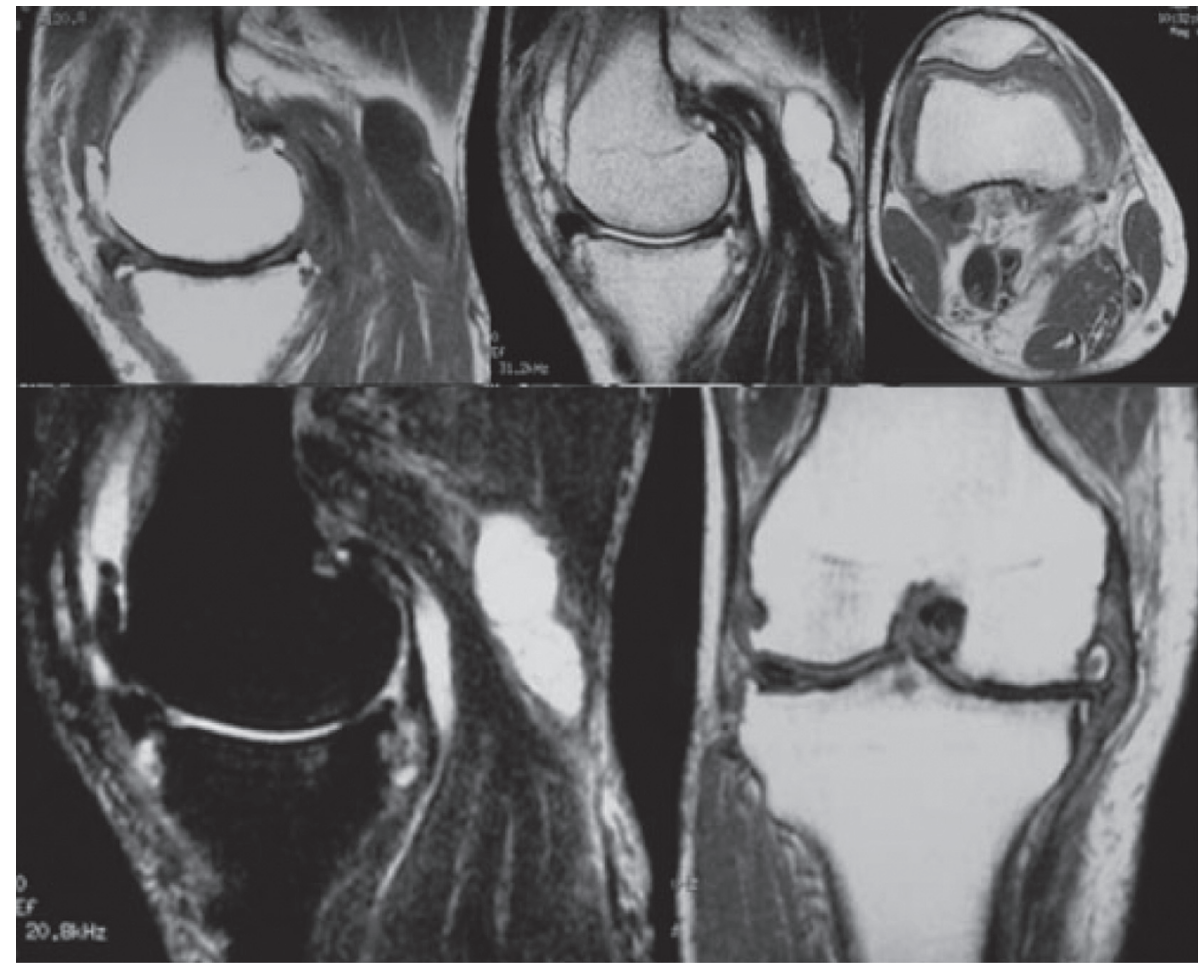

FIgURE 3: Part of the training sample diagram. 


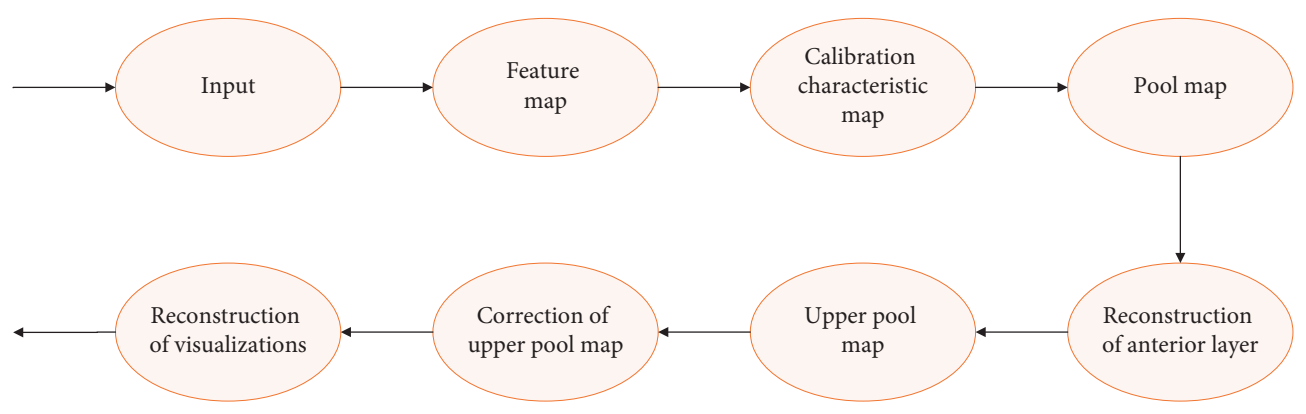

Figure 4: Schematic diagram of deconvolution neural network structure.

addition, the left was a deconvolutional network layer that connected to a convolutional layer.

The main research content of deconvolution neural network visualization [16] has three types, including depooling, deactivation, and deconvolution. The working principle of $\mathrm{CNN}$ is to adjust parameter variables through training and learn features layer by layer to extract image features. In this experiment, it was attempted to extract the feature graph from the network model layer by layer to make the feature graph visible.

Figure 5 indicated that the convolutional layer of the first unit extracted some low-level image features such as basic gray color and edge information. Figure 6 meant that the feature extraction of the convolutional layer of the second unit was more complicated, and CNN began to learn some texture features and more details. Figure 7 revealed that the features extracted by the convolutional layer of the third unit were more discriminative, each output feature map was different, and CNN learned discriminative features [17]. It was found that the image features were more distinguishable after being learned by the CNN model, and the visual feature maps extracted by the network could be fed back to the clinician, which had important research value for the doctor's clinical diagnosis.

2.5. Judgment Criteria. According to the Ribbens diagnostic criteria, the anechoic zone thickness of the proximal joint space $\geq 1 \mathrm{~mm}$ was judged as the presence of joint effusion.

According to Walther standard, synovial membrane thickness $\geq 2 \mathrm{~mm}$ was regarded as synovial thickening.

The judgment criteria for Cortical bone erosion and defect were as follows: cortical bone erosion is $\leq 2 \mathrm{~mm}$; cortical bone defect is $>2 \mathrm{~mm}$.

VAS was used for pain assessment, ranging from 0 to 10 points. 0 point meant no pain, and 10 points indicated the most severe pain that was unbearable (Figure 8).

2.6. Statistical Analysis. SPSS 24.0 statistical software was adopted for statistical analysis of the data of this study. Measurement data were expressed as $\bar{x} \pm s, \chi^{2}$ test was for the count data comparison, and Pearson rank correlation analysis method was employed to analyze the correlation between MRI and DR ratings. In addition, $P<0.05$ indicated that the difference was statistically substantial.

\section{Results}

3.1. The Imaging Manifestations of Normal Knee Joints. A 47year-old male volunteer from the healthy control group showed the good joint condition of both knees with no abnormality after imaging examination. The imaging images of the unaffected knee joint mainly showed the following aspects (Figure 9). First, the synovial sac of the knee and the tendons of the quadriceps femoris were observed from the front, and the synovial sac showed no effusion and no synovial hyperplasia. Second, the medial region was observed, the bone of the lower femur and tibia were normal, and the bone was continuous and smooth. Third, the meniscus in the medial and lateral regions was triangular and regular in shape, the medial and lateral collateral ligaments were in the normal echo zone, and the bone was in the obvious strong echo zone. Fourth, there was no effusion in the popliteal fossa in the dorsal zone.

3.2. MRI Image Evaluation Based on CNN Model. A 50-yearold male patient in the lesion group had localized pain in both knee joints and had soft legs for more than 2 months and was clinically diagnosed as having meniscus injury (Figure 10(a)). A female patient from the lesion group, aged 48 years old, had acidness and distension of the left knee joint and local pain for more than 11 months, which was clinically diagnosed as intracapsular lesions (Figure 10(b)). Therefore, Figure 10 indicated that the MRI image analyzed by the CNN model had a more obvious display rate for the nonbone changes of osteoarthropathy, such as joint capsule inner disease, knee cartilage degeneration, knee joint effusion, osteoarthritis, and cartilage injury, than DR images.

3.3. Traditional DR Image Evaluation. In the lesion group, a 61-year-old female patient had a persistent dull pain in both knee joints and stiffness for more than 5 months and was clinically diagnosed with hyperosteogeny (Figure 11(a)). A patient, female, 70 years old, with local swelling and pain in the right knee joint, joint stiffness for more than 1 year, and slight deformity for more than 3 months, was clinically diagnosed as having lacunar stenosis of the right knee joint (Figure 11(b)). Furthermore, Figure 12 revealed that the display rate of DR images for bone diseases such as 


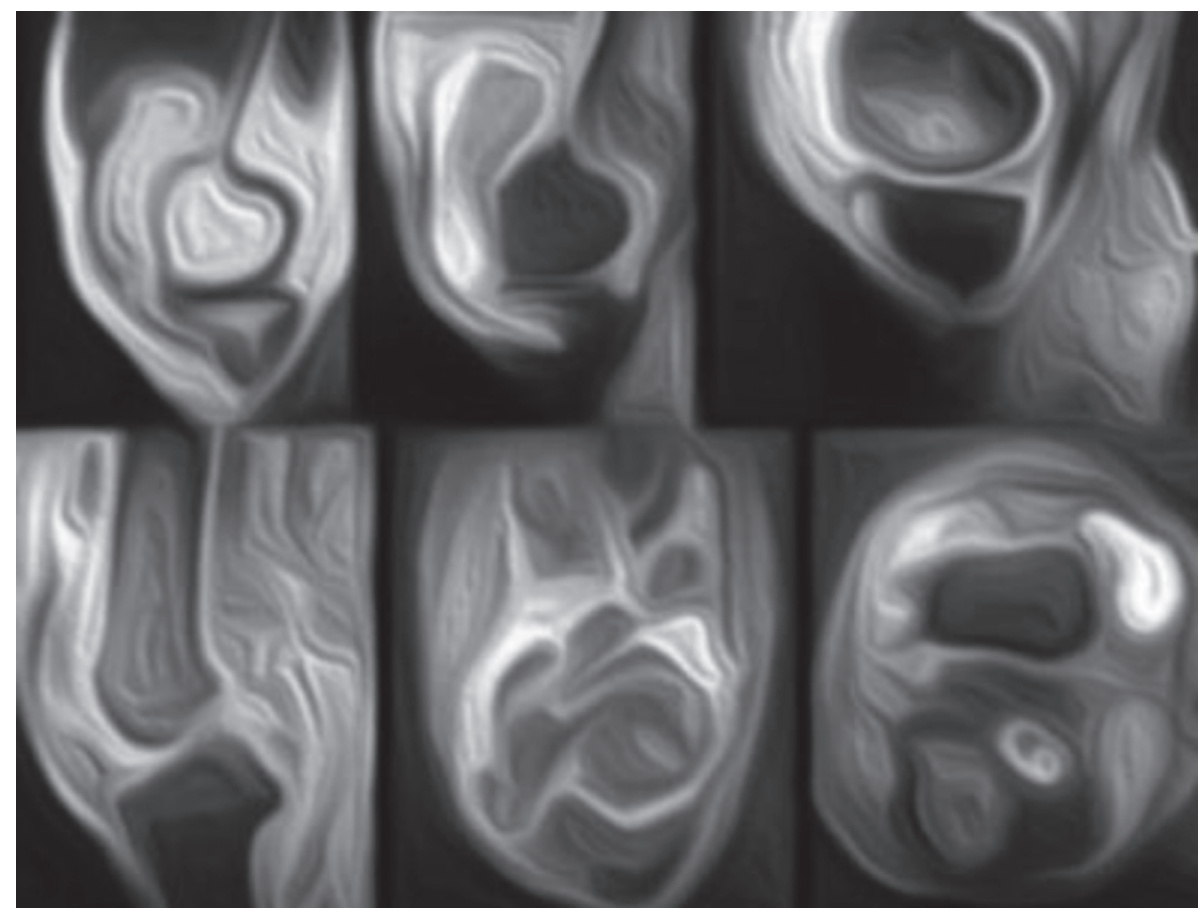

FIgURE 5: The images after the processing by the first unit convolutional layer.

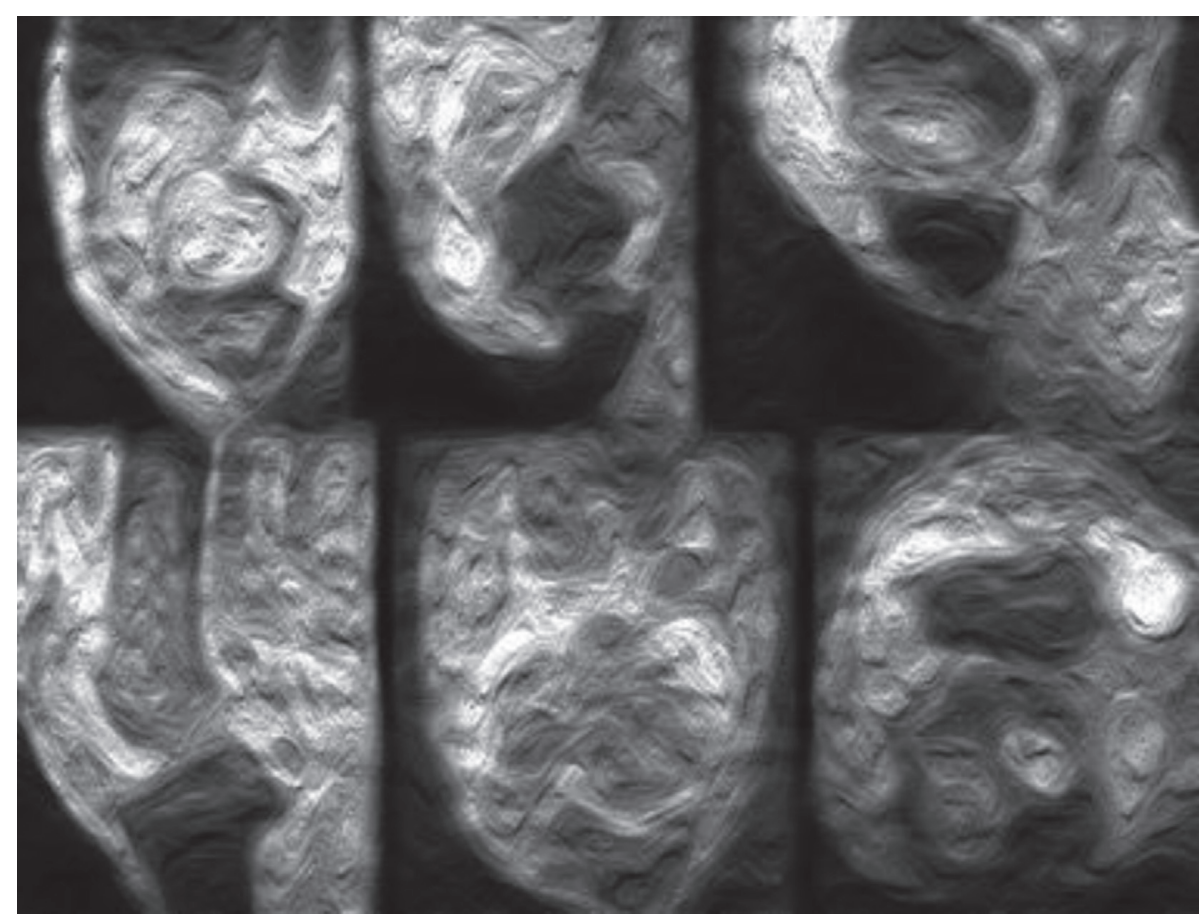

Figure 6: The images after the processing by the second unit convolutional layer.

proliferative osteoarthropathy and knee joint space stenosis was slightly higher than the rate of MRI, but the difference was not big.

3.3.1. Correlation between DR Image Rating and Clinical Manifestations. The correlation coefficient between the DR image rating ( $0-4$ grade) and the VAS scoring method was
0.713, which was statistically remarkable $(P<0.01)$ (Figure 13).

3.3.2. Correlation between MRI Image Rating Analyzed by CNN Model and Clinical Manifestations. The correlation coefficient between the MRI image rating ( $0-4$ grade) and the VAS scores analyzed by the CNN model was 0.865 , 


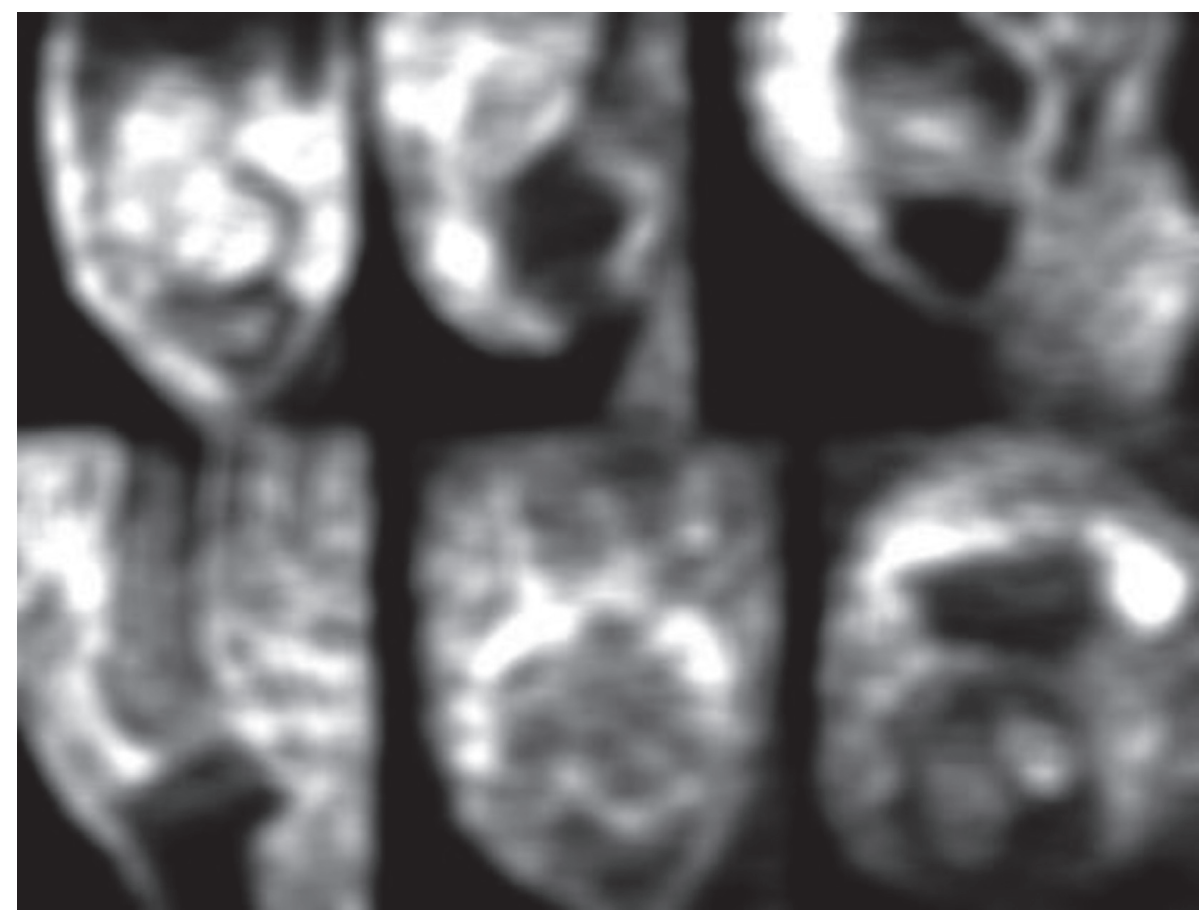

FIGURE 7: The images after the processing by the third unit convolutional layer.
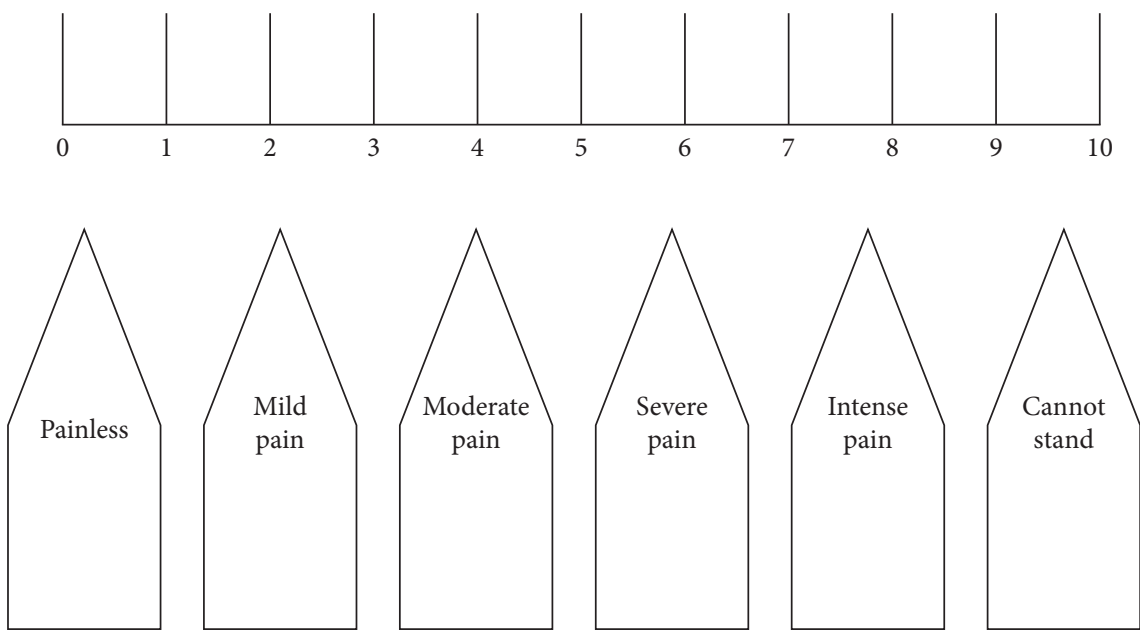

FIgURE 8: VAS scoring method.

showing a statistically marked meaning $(P<0.01)$ (Figure 14).

\subsubsection{The Correlation between the MRI Image Analyzed by} the CNN Model and the DR Image Rating. The correlation coefficient between the MRI rating image analyzed by the $\mathrm{CNN}$ model and the DR image rating was 0.866 , so there was a statistical meaning $(P<0.01)$. For cases with more severe lesions, the MRI images analyzed by the CNN model were more consistent with the DR image rating. For cases with milder lesions, the evaluation results of the MRI images analyzed by the CNN model were better than the DR images, as shown in Figure 15.

\section{Discussion}

The knee joint is the joint with the most synovium, the largest articular surface, and the most complex structure in the human body, and also the joint with the largest load in the human body. In daily life and sports, cartilage tissue injury and ligament sprain often occur, which may lead to some bone diseases with time [18]. At present, imaging has been the first choice for pathological diagnosis. However, it has a high requirement for manual image reading, and manual image data processing will also increase the time cost and affect the quality of pathological analysis and diagnosis. In recent years, artificial intelligence has played a significant role in assisting the diagnosis of clinicopathological imaging. 


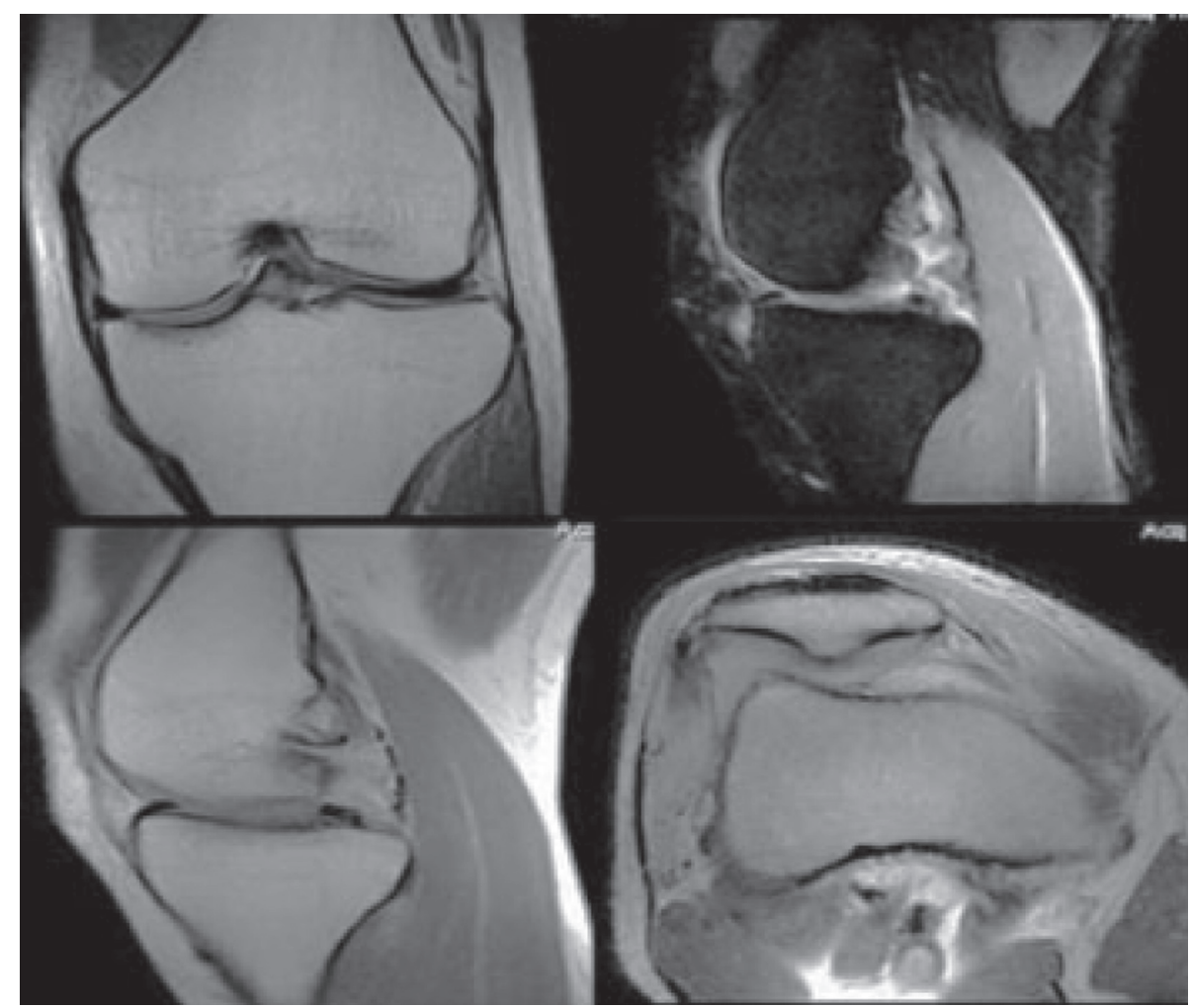

FIGURE 9: Images of normal knee joint.

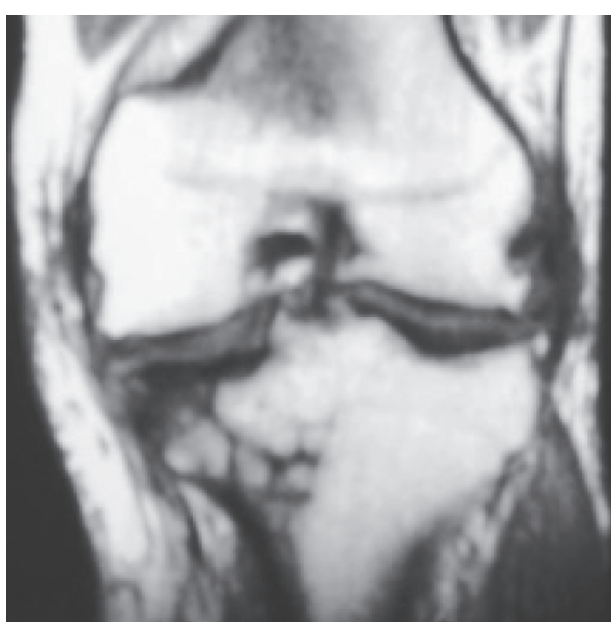

(a)

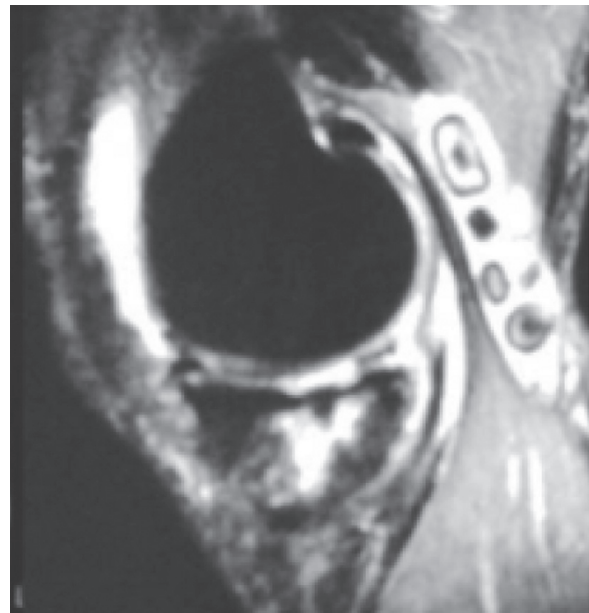

(b)

FIgURE 10: MRI images of meniscus injury (a) and joint capsule inner layer disease (b) based on CNN model.

$\mathrm{CNN}$ is a representative model of $\mathrm{DL}$, which can reduce the complexity of computer learning, convolve images in sequence, and extract microscopic histological features. In 2020, Chen et al. [19] also pointed out that deep learning models were increasingly widely applied in images. Therefore, the network model was established in this study based on the CNN algorithm, which was adopted to analyze MRI images and diagnose diseases. Firstly, MRI images were collected from 100 patients with sports knee osteoarthropathy and 50 healthy subjects, which were used for prediagnostic training, and a series of pretreatments were performed on the sample images. Then, a vertical and horizontal array was stored, and the corresponding binary classification label was stored in a data storage file and read into the network. After verification, 130 correct rates were obtained, with an average accuracy of $86.67 \%$. Then, the image of the patient was read into the $\mathrm{CNN}$ model for the diagnosis of the disease, and the predicted result of the disease was "the patient was suspected to have sports knee osteoarthropathy," so the diagnosis was correct. This 


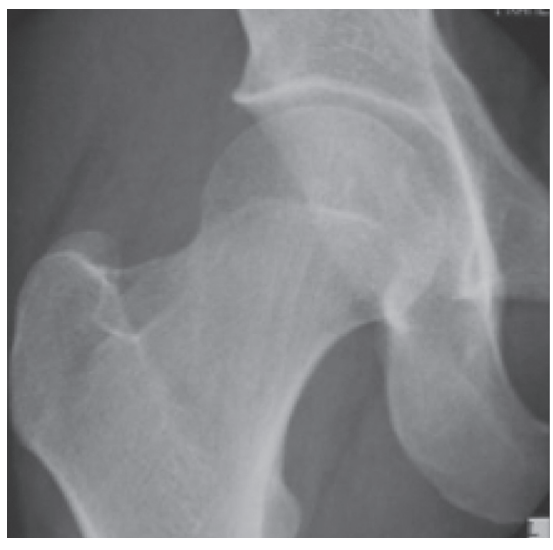

(a)

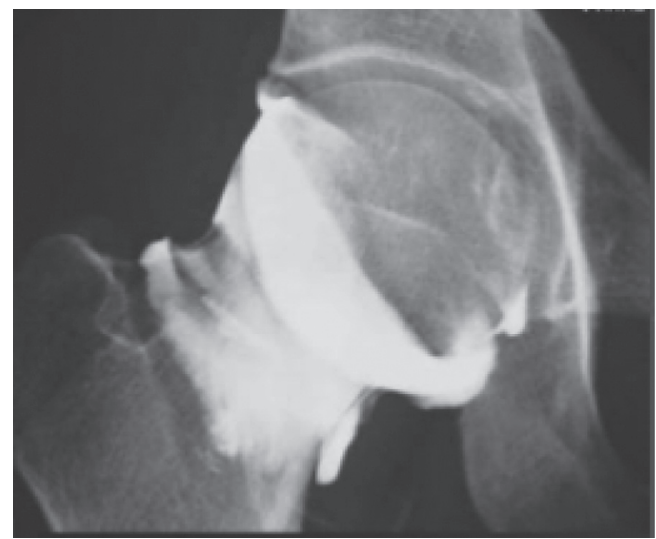

(b)

FIGURE 11: DR images of bone hyperplasia (a) and knee joint space stenosis (b).

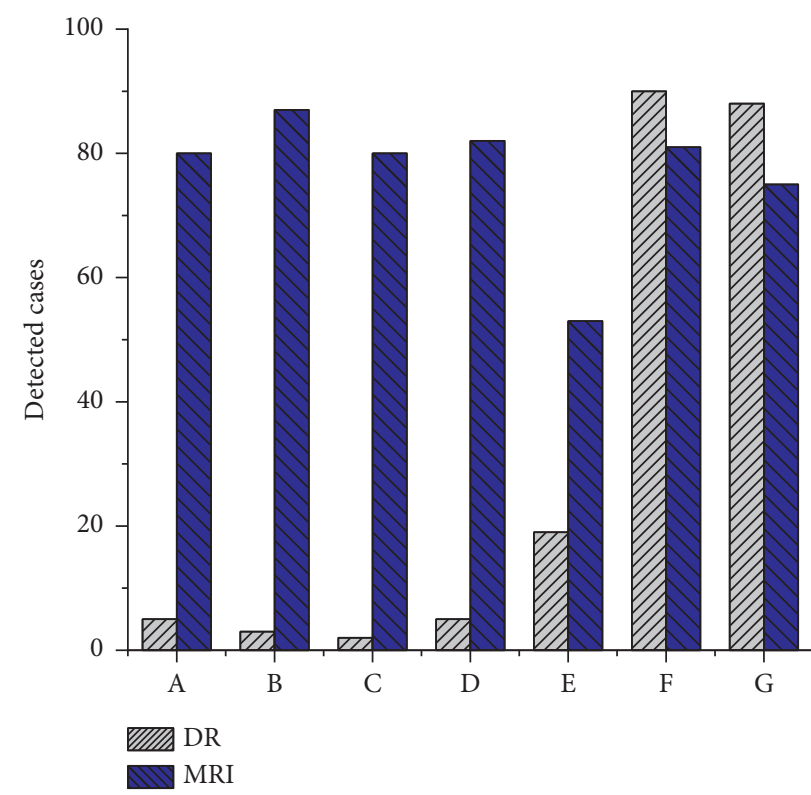

FIGURE 12: Comparison of the number of cases shown by DR and MRI images. (Note: A: joint capsule inner layer disease; B: knee cartilage degeneration; C: knee joint effusion; D: osteoarthritis; E: cartilage injury, F: proliferative osteoarthropathy; G: knee joint space narrowing).

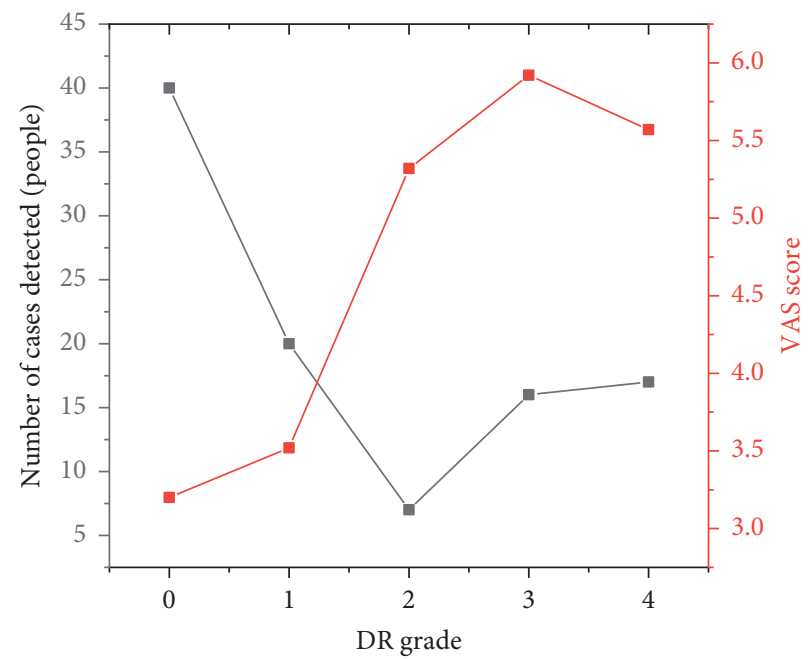

FIGURE 13: Correlation between DR image rating and clinical manifestations. 


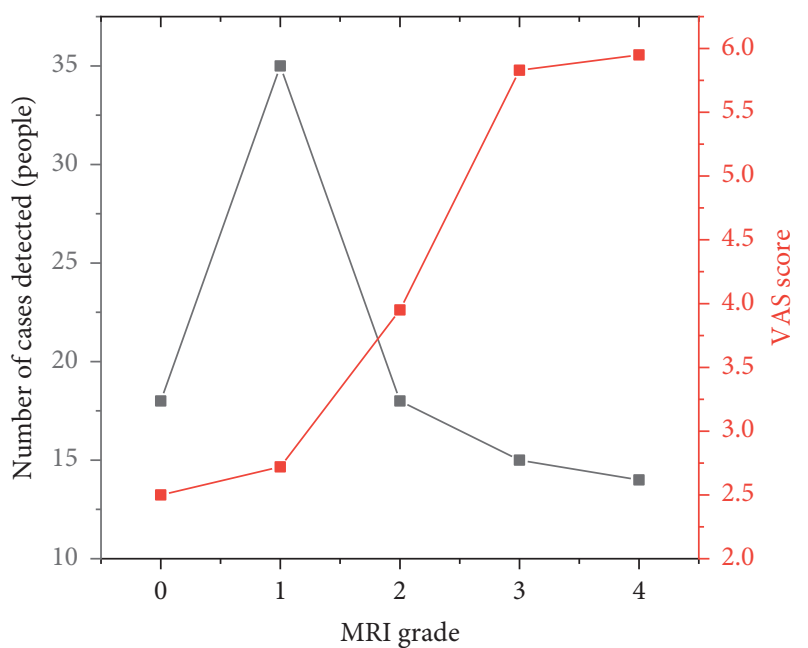

FIGURE 14: Correlation between MRI image rating analyzed by CNN model and clinical manifestations.

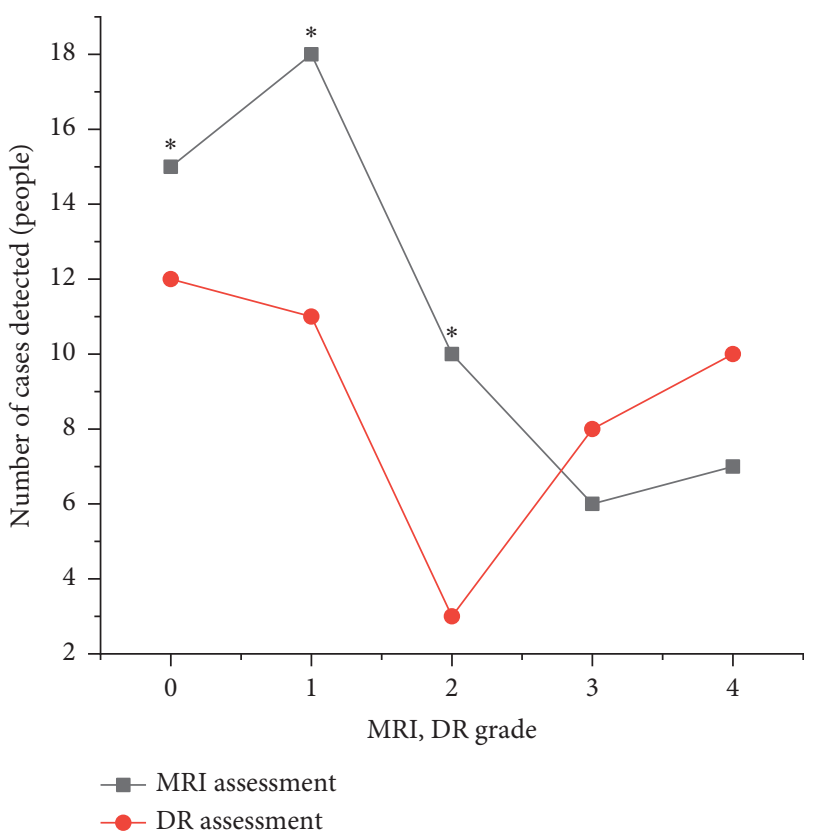

FIGURE 15: The correlation between the MRI image analyzed by the $\mathrm{CNN}$ model and the DR image rating. (Note: $*$ indicated that there was a difference between the evaluation result of the MRI image and the DR image $(P<0.05)$.

indicated that the accuracy and performance of the $\mathrm{CNN}$ model were better compared with computer-assisted diagnosis. For all patients with traditional DR check by MRI, a contrast of image display, the experimental results suggested that the CNN model analysis of MRI images of some nonosseous changes in disease rate of DR images had obvious advantages; its image rating and VAS score of correlation coefficient was 0.865 , higher than $\mathrm{DR}$, with a statistical significance $(P<0.01)$. For cases with mild lesions, the evaluation results of MRI images analyzed by the CNN model were also significantly better than those of DR images. This result was in line with the research conclusion of Liu et al. [20]. Thus, it suggested that the CNN algorithm could detect classified MRI images well, automatically analyze and extract effective information for parallel quantitative calculation, improve precision and accuracy, reduce the probability of misdiagnosis, improve work efficiency, decrease working hours, reduce the burden of doctors, and avoid some quality problems of analysis and diagnosis. It was of great significance for the diagnosis of clinicopathological imaging.

\section{Conclusion}

In this experiment, the network model was constructed based on the CNN algorithm to be used in MRI examinations. 100 patients with sports knee osteoarthropathy and 50 healthy subjects were selected for experimental research, and the analyzed MRI images were compared with traditional DR images. The results found that the MRI images analyzed by the CNN model showed high accuracy and display rate for some nonbone change diseases, which was superior to the traditional digital X-ray photography, and could be used as a more reliable computer network model for clinical diagnosis. However, the number of cases included in this experiment is not large enough, and the results may also be affected by the subjective judgment of the clinician and the patient's factors. The follow-up consideration is to increase the sample size of sports knee osteoarthropathy patients, and a variety of examination methods are cooperated and applied in this study. The clinical application of the CNN algorithm is not long and needs further improvement and promotion. It is believed that, with the rapid development of science and technology, the application in imaging examinations of sports knee osteoarthropathy will become more and more extensive, providing more clinical evidence.

\section{Data Availability}

No data were used to support this study.

\section{Conflicts of Interest}

The author declares that there are no conflicts of interest.

\section{References}

[1] M. J. Lespasio, N. S. Piuzzi, M. E. Husni, G. F. Muschler, A. Guarino, and M. A. Mont, "Knee osteoarthritis: a primer," The Permanente Journal, vol. 21, pp. 16-183, 2017.

[2] H. Madry, E. Kon, V. Condello et al., "Early osteoarthritis of the knee," Knee Surgery, Sports Traumatology, Arthroscopy, vol. 24, no. 6, pp. 1753-1762, 2016.

[3] X. H. Meng, Z. Wang, X. N. Zhang, J. Xu, and Y. C. Hu, "Rheumatoid arthritis of knee joints: MRI-pathological correlation," Orthopaedic Surgery, vol. 10, no. 3, pp. 247-254, 2018.

[4] F. P. Luyten, Z. S. Bierma, F. Dell'Accio et al., "Toward classification criteria for early osteoarthritis of the knee," Seminars in Arthritis and Rheumatism, vol. 47, no. 4, pp. 457-463, 2018. 
[5] M. Shujaat, A. Wahab, H. Tayara, and K. T. Chong, "pcPromoter-CNN: a CNN-based prediction and classification of promoters," Genes, vol. 11, no. 12, p. 1529, 2020.

[6] X. Zhao, S. Qi, B. Zhang et al., "Deep CNN models for pulmonary nodule classification: model modification, model integration, and transfer learning," Journal of X-Ray Science and Technology, vol. 27, no. 4, pp. 615-629, 2019.

[7] J. Chen, S. Bi, G. Zhang, and G. Cao, "High-density surface EMG-based gesture recognition using a $3 \mathrm{D}$ convolutional neural network," Sensors, vol. 20, no. 4, p. 1201, 2020.

[8] F. Schwendicke, T. Golla, M. Dreher, and J. Krois, "Convolutional neural networks for dental image diagnostics: a scoping review," Journal of Dental Medicine, vol. 91, Article ID 103226, 2019.

[9] F. Su, H. Y. Shang, and J. Y. Wang, "Low-rank deep convolutional neural network for multitask learning," Computational Intelligence and Neuroscience, vol. 2019, Article ID 7410701, 10 pages, 2019.

[10] S. Biswas, H. K. Aggarwal, and M. Jacob, "Dynamic MRI using model-based deep learning and SToRM priors: MoDLSToRM," Magnetic Resonance in Medicine, vol. 82, no. 1, pp. 485-494, 2019.

[11] D. Wang, F. Tian, S. X. Yang, Z. Zhu, D. Jiang, and B. Cai, "Improved deep CNN with parameter initialization for data analysis of near-infrared spectroscopy sensors," Sensors, vol. 20 , no. 3 , p. $874,2020$.

[12] Y. Y. Baydilli and Ü. Atila, "Classification of white blood cells using capsule networks," Computerized Medical Imaging and Graphics, vol. 80, Article ID 101699, 2020.

[13] R. Bhalodia, S. Y. Elhabian, L. Kavan, and R. T. Whitaker, "A cooperative autoencoder for population-based regularization of CNN image registration," Medical Image Computing and Computer-Assisted Intervention, vol. 11765, pp. 391-400, 2019.

[14] Y. G. Kim, S. M. Lee, K. H. Lee, R. Jang, J. B. Seo, and N. Kim, "Optimal matrix size of chest radiographs for computer-aided detection on lung nodule or mass with deep learning," $E u$ ropean Radiology, vol. 30, no. 9, pp. 4943-4951, 2020.

[15] A. Shajkofci and M. Liebling, "Spatially-variant CNN-based point spread function estimation for blind deconvolution and depth estimation in optical microscopy," IEEE Transactions on Image Processing, vol. 29, 2020.

[16] A. M. Mota, M. J. Clarkson, P. Almeida, and N. Matela, “An enhanced visualization of DBT imaging using blind deconvolution and total variation minimization regularization," IEEE Transactions on Medical Imaging, vol. 39, no. 12, pp. 4094-4101, 2020.

[17] R. Yamashita, M. Nishio, R. K. G. Do, and K. Togashi, "Convolutional neural networks: an overview and application in radiology," Insights Imaging, vol. 9, no. 4, pp. 611-629, 2018.

[18] V. Musahl, E. Herbst, J. M. Burnham, and F. H. Fu, "The anterolateral complex and anterolateral ligament of the knee," Journal of the American Academy of Orthopaedic Surgeons, vol. 26, no. 8, pp. 261-267, 2018.

[19] Y. Chen, S. J. Hu, H. Mao, W. Deng, and X. Gao, "Application of the best evacuation model of deep learning in the design of public structures," Image and Vision Computing, vol. 102, Article ID 103975, 2020.

[20] F. Liu, R. Kijowski, L. Feng, and G. El Fakhri, "High-performance rapid MR parameter mapping using model-based deep adversarial learning," Magnetic Resonance in Imaging, vol. 74, pp. 152-160, 2020. 